\title{
Precise Indoor Localization Using Smart Phones
}

\author{
Eladio Martin \\ Dept. of EECS \\ University of \\ California, Berkeley \\ Berkeley, CA 94720 \\ emartin@berkeley.edu
}

\author{
Oriol Vinyals \\ International Computer \\ Science Institute \\ 1947 Center Street, \\ Berkeley, CA 94704-1198 \\ vinyals@icsi.berkeley.edu
}

\author{
Gerald Friedland \\ International Computer \\ Science Institute \\ 1947 Center Street, \\ Berkeley, CA 94704-1198 \\ fractor@icsi.berkeley.edu
}

\author{
Ruzena Bajcsy \\ Dept. of EECS \\ University of California, \\ Berkeley \\ Berkeley, CA 94720 \\ bajcsy@eecs.berkeley.edu
}

\begin{abstract}
We present an indoor localization application leveraging the sensing capabilities of current state of the art smart phones. To the best of our knowledge, our application is the first one to be implemented in smart phones and integrating both offline and online phases of fingerprinting, delivering an accuracy of up to 1.5 meters. In particular, we have studied the possibilities offered by WiFi radio, cellular communications radio, accelerometer and magnetometer, already embedded in smart phones, with the intention to build a multimodal solution for localization. We have also implemented a new approach for the statistical processing of radio signal strengths, showing that it can outperform existing deterministic techniques.
\end{abstract}

\section{Categories and Subject Descriptors}

D.2.2 [Software Engineering]: Design Tools and Techniques Decision tables, Modules and interfaces.

\section{General Terms}

Algorithms, Measurement, Experimentation.

\section{Keywords}

Indoor Localization, Smart Phones.

\section{INTRODUCTION}

With the trend towards ubiquitous computing, context awareness is becoming a key factor in multiple applications, with location information playing a fundamental role. Most recent studies to monitor location with smart phones make use of the embedded GPS radio and accelerometers [1]. However, the location information obtained through GPS is only reliable in certain outdoor environments with direct visibility to the satellites, while the accelerometer data are generally too noisy to obtain accurate location information. Reference [2] shows the importance of indoor localization, especially for hospitals and government offices.

Permission to make digital or hard copies of all or part of this work for personal or classroom use is granted without fee provided that copies are not made or distributed for profit or commercial advantage and that copies bear this notice and the full citation on the first page. To copy otherwise, or republish, to post on servers or to redistribute to lists, requires prior specific permission and/or a fee.

MM'10, October 25-29, 2010, Firenze, Italy.

Copyright 2010 ACM 978-1-60558-933-6/10/10...\$10.00.
Within the emerging market of smart phones, Android Operating System is well suited for modern devices and offers an excellent option for localization due to its support of diverse sensors and hardware which can be leveraged for localization beyond GPS (e.g. WiFi, cellular communications, accelerometer), and its higher efficiency in comparison with other platforms such as J2ME [7]. In this sense, recent research work [8] presents Location Based Services running on Android, but showing the need of a precise indoor localization system, since most of these applications rely on GPS.

We have carried out a detailed study of the possibilities offered by 3 available resources in smart phones: WiFi radio, cellular communications radio and accelerometer, with the intention to build a multimodal approach for localization in Android smart phones.

To the best of our knowledge, our application represents the first approach for indoor localization making use of smart phones. In Section 2 the related work in this area is summarized, while in Section 3, technical details about our solution are presented, gathering the conclusions in Section 4.

\section{RELATED WORK}

Radio Signal Strength Indications (RSSI) can be translated into distances from beacon points by means of theoretical or empirical radio propagation models. The following expression accounts for a general radio propagation model delivering the received power $P_{r}$ :

$$
P_{r}=P_{t}\left(\frac{\lambda}{4 \pi d}\right)^{n} G_{t} G_{r}
$$

Where $P_{t}$ represents the transmitted power, $\lambda$ the wavelength of the radio signal, $G_{t}$ and $G_{r}$ the gains of the transmitter and receiver antennas respectively, $d$ the distance separating them, and $n$ is the path loss coefficient, typically ranging from 2 to 6 depending on the environment. The two main approaches for the estimation of location making use of RSSI values are: 1) "fingerprinting", where a pre-recorded radio map of the area of interest is leveraged to infer locations through best matching, and 2) "propagation based", in which RSSI values are used to calculate distances through the computation of the path loss. "Propagation based" techniques can face errors of up to $50 \%$ [8] due to multipath, non line-of-sight conditions, interferences and other shadowing effects, rendering this technique unreliable and inaccurate, especially for indoor environments, where multipath is very important. Several authors have tried to improve the efficiency of this technique for indoor environments, introducing 
new factors in the path loss model to account for wall attenuation, multipath or noise [9], but the hardware and software requirements due to the complexity of the method and the overall poor accuracy achieved makes this approach unfeasible for current state of the art smart phones. On the other hand, fingerprinting techniques have already proved to be able to deliver better accuracies [10]. For example, the Intelligent Service Integration Laboratory in the Korea Advanced Institute of Science and Technology is developing a remarkable system called Elekspot to register indoor sites with associated information using WiFi fingerprints. In these techniques, the mobile terminal estimates its location through best matching between the measured radio signals and those corresponding to locations previously registered in the radio map. This process consists of two stages:

1) Training phase, also called offline phase, in which a radio map of the area in study is built. RSSI values from different beacons are recorded at different locations; the separation between these chosen locations will depend on the area in study, and for instance, for indoor environments this separation can be of around a meter [11]. Each measurement consists of several readings, one for each radio source in range [12].

2) Online phase, in which the mobile terminal infers its location through best matching between the radio signals being received and those previously recorded in the radio map. Localization algorithms employed in this case generally make use of deterministic or probabilistic techniques:

Deterministic techniques store scalar values of averaged RSSI measurements from the access points [13]. The most relevant techniques in this group are:

a) "Closest point", or "nearest neighbor in signal space" [14].

b) "Nearest neighbor in signal space-average" [13], [15], choosing $\mathrm{k}$ nearest neighbors and calculating the centroid of that set.

c) "Smallest polygon", selecting several nearest neighbors which will form various polygons, and the centroid of the smallest polygon will be considered as the estimated location [13].

Probabilistic techniques choose the location from the radio map as the one with the highest probabilities, and usually require the storage of RSSI distributions from the different beacons at each location in the radio map [14].

Fingerprinting techniques are especially appropriate for the range of frequencies in which GSM and WiFi networks operate (aprox. $850 \mathrm{MHz}$ to $2.4 \mathrm{GHz}$ ) because of two main reasons [12]: the signal strength at those frequencies presents an important spatial variability, and also a reliably consistency in time (despite the variable nature of radio signals).

Considering GSM as an example for cellular communications technology, although it makes use of power control both at the mobile terminal and base station, the data on the Broadcast Control Channel $(\mathrm{BCCH})$ is transmitted at full and constant power, making this channel suitable for fingerprinting [12]. Several authors have tried this approach for localization, but with the need of dedicated and complex hardware. In order to improve the accuracy of this approach, a selection among all the measured signals is recommended, rejecting those which are either too noisy, too stable across all areas or simply do not provide enough information [11].
Regarding WiFi technology, several research groups have already tried to leverage RSSI fingerprinting for localization:

- Radar [16]: represents the first fingerprinting system achieving the localization of portable devices, with accuracies of 2 to 3 meters.

- Horus [17]: based on the Radar system, it manages a performance improvement making use of probabilistic analysis.

- Compass [18]: applies probabilistic methods and leverages object orientation to improve precision, claiming errors below 1.65 meters.

- Ekahau [19]: commercial solution using $802.11 \mathrm{~b} / \mathrm{g}$ networks, achieving precisions from 1 to 3 meters in normal conditions.

Nevertheless, all the existing approaches use dedicated and complex hardware, making them unfeasible for direct implementation in current state of the art smart phones. To the best of our knowledge, our indoor localization application is the first one to accurately leverage RSSI fingerprinting in smart phones.

Besides cellular communications and WiFi technologies, the RSSI fingerprinting technique for localization can be utilized with other radiofrequency technologies including:

- Bluetooth, which despite the extra infrastructure requirements in comparison with $\mathrm{WiFi}$, it can achieve accuracies in the range of 1.2 meters.

- Conventional radio, can also be used for localization. However, the requirement of dedicated hardware and the fact that devices can be located only down to a suburb, represent important drawbacks.

- Digital TV signals have also proved to be suitable for localization, but subject to dedicated hardware requirements and low resolutions.

- Zigbee technology can also be applied for localization through fingerprinting [20], achieving accuracies of approximately 2 meters. However, this technology also requires extra hardware for a correct implementation, constituting a major drawback.

\section{TECHNICAL DETAILS OF OUR LOCALIZATION APPLICATION}

We have studied the possibilities offered by 3 available resources in smart phones: WiFi radio, cellular communications radio and accelerometer, with the aim to build a multimodal approach for localization. As will be explained later in this Section, the consideration of WiFi radios represents the most reliable approach for indoor localization in our experimental setup in buildings across the University of California, Berkeley campus. In fact, RSSI information from WiFi beacons deployed within buildings allows us to obtain a radio map of different locations (technique called fingerprinting), and we will estimate locations through the comparison of the current RSSI measurements with those stored in the radio map. Different attempts to obtain RSSI-based indoor localization without fingerprinting show an important loss of accuracy [5]. Also, many fingerprinting-based localization systems make use of dedicated hardware for the collection of data 
in the training phase, while in the measurement phase, the actual mobile device used for localization is different, resulting in an error called "signal reception bias" [6], due to the differences in antennas characteristics and measurement acquisitions schemes between different equipments. In fact, we have carried out tests showing a difference of approximately $10 \mathrm{~dB}$ in average between RSSI values measured with a Dell Latitude laptop and those measured with a Motorola Droid cell-phone. Consequently, we have integrated both the training and measurement phases in fingerprinting within the same mobile device. To the best of our knowledge, our application is the first one following this approach with smart phones. Moreover, the way the fingerprints are taken in the training phase should reproduce as accurately as possible the way the measurements will be carried out in the localization phase. In this sense, the orientation of the phone (obtainable from accelerometer and magnetometer data) helps enhance the localization accuracy. And in order to minimize errors due to human body effect [7], the cell-phone should be handled during the training phase as close as possible to the normal conditions in which it will be used in the measurement phase.

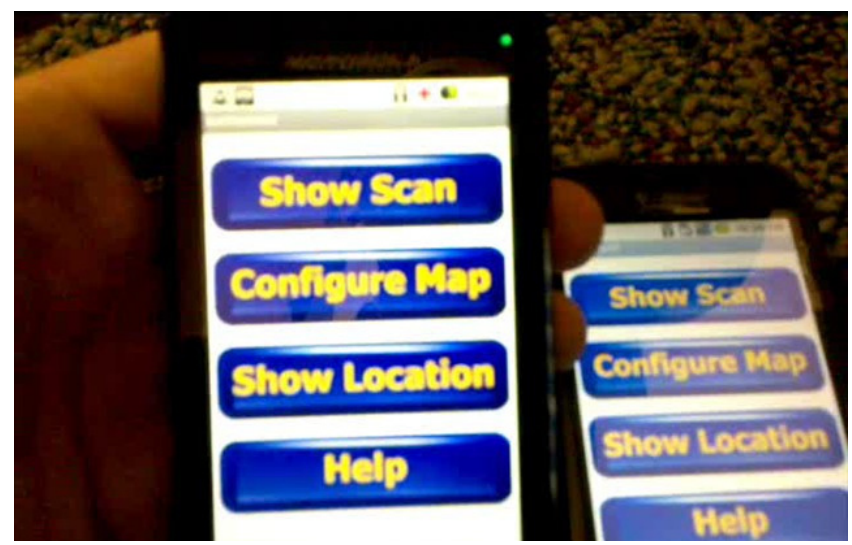

Figure 1. Main interface of our Localization application in the Droid (left) and G1 (right) phones.

\subsection{Experimental Setup}

We have carried out tests to measure different radiofrequency signal strengths within the Cory building in the University of California, Berkeley campus. As will be explained further in this Section, WiFi technology offers the most reliable approach for indoor localization in our building, because of the important deployed infrastructure of WiFi Access Points providing coverage in the whole building. For the measurement of the signals and practical implementation of our localization application, we have used smart phones running on Android, in particular the G1 and the Droid. The sensitivity of these Android phones ranges from $45 \mathrm{dBm}$ to $-104 \mathrm{dBm}$. Nevertheless, values below $-85 \mathrm{dBm}$ are generally too inconsistent to be leveraged as reference. Consequently, RSSI values above $-80 \mathrm{dBm}$ are desirable in order to obtain reliable results. Processing this information statistically, we have built an Android application for localization, and we have tested it in locations where $25 \mathrm{WiFi}$ radios in average were listened (approximately $40 \%$ of them with RSSI above $-80 \mathrm{dBm}$ ), obtaining accuracies in the order of 1.5 meters even within a same room, and with real-time dynamicity (refreshment of location information every second). However, we have found a major challenge with some smart phones (early generations of smart phones regardless of their Operating System), in the sense that the RSSI values were not refreshed dynamically. Smart phones running on Android 2.0 and above do not have this issue. It must also be noticed that as the number of WiFi radios (and their RSSI values) that can be listened in a specific location decreases (e.g. only $2 \mathrm{WiFi}$ radios with RSSI values above $-75 \mathrm{dBm}$ ), our application's localization accuracy drops, to the point of obtaining only room accuracy.

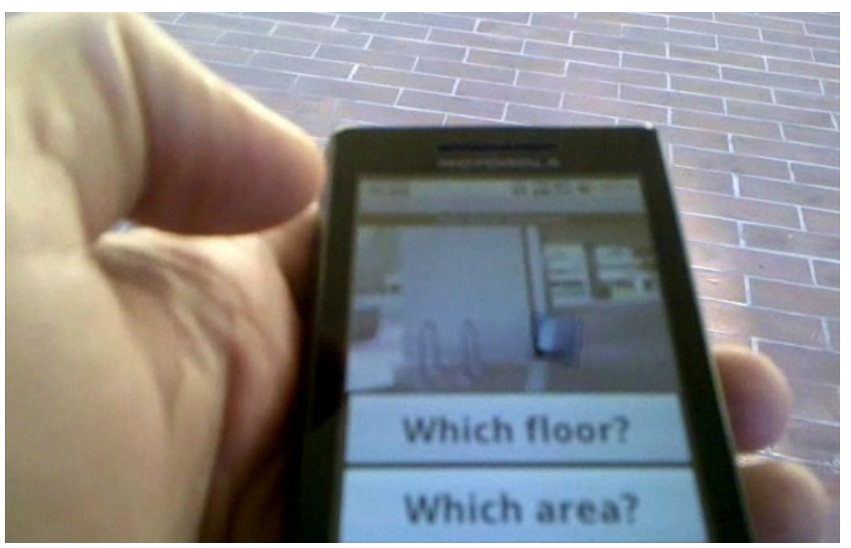

Figure 2. Localization Application in the Droid showing location information as multimedia messages.

In our experimental setup, each WiFi Access Point has 5 radios (each represented by a MAC address). For example, 00:23:04:89:db:20, 00:23:04:89:db:21, 00:23:04:89:db:22, 00:23:04:89:db:25 and 00:23:04:89:db:26 are 5 radios belonging to the same Access Point. RSSI values (in $\mathrm{dBm}$ ) from the same Access Point can show important standard deviations in between consecutive scans (within the same radio) and also in between different radios within the same Access Point. Consequently, averaging of values both within the same Access Point and over time provides much more stable values that can successfully be used as a fingerprint component of each particular location. We call this approach "Nearest Neighbor in signal space and Access Point averages", and the results summarized in Table 1 show that our approach can outperform existing deterministic techniques (the resolution metric, in percentage, accounts for the number of true positives obtained during localization tests):

Table 1. Comparison of accuracies of different approaches in terms of success in location estimation

\begin{tabular}{|c|c|c|c|}
\hline \multirow{2}{*}{ Technique } & \multicolumn{3}{|c|}{ Resolution (\% of success) } \\
\cline { 2 - 4 } & Room & $\mathbf{2}$ meters & 1 meter \\
\hline Closest Point & $85 \%$ & $39 \%$ & $18 \%$ \\
\hline $\begin{array}{c}\text { Nearest Neighbor in Signal } \\
\text { Average }\end{array}$ & $78 \%$ & $39 \%$ & $26 \%$ \\
\hline $\begin{array}{c}\text { Smallest Polygon } \\
\text { Nearest Neighbor in Signal } \\
\text { and Access Point averages }\end{array}$ & $84 \%$ & $45 \%$ & $26 \%$ \\
\hline \multicolumn{2}{|c|}{} & $48 \%$ & $32 \%$ \\
\hline
\end{tabular}

It must also be noticed that there are radios which only show up at some particular locations, but with a very low RSSI value. For instance, 2a:1e:af:0e:04:15 could only be noticed in a small area within a room in our building with $-97 \mathrm{dBm}$. Nevertheless, this 
information with so low RSSI values can be considered as noise, and therefore it is not reliable to use radios with RSSI below -85 $\mathrm{dBm}$ as a disambiguation factor. The useful information to disambiguate locations can be found at RSSI values above -85 $\mathrm{dBm}$, which in our case corresponds to approximately 3 Access Points with stable enough values to allow us to correctly distinguish between the different locations.

RSSI information from cellular base stations could theoretically be used to disambiguate locations for which the WiFi radio map offers doubts. Nevertheless, we have found this approach unfeasible with current state of the art smart phones, because the refreshment rate of RSSI values is very slow (not dynamic enough for indoor walking) and the granularity in the RSSI values is poor and hardware dependent (e.g. G1 only distinguishes between 4 bars of coverage, and Droid only provides a few more intermediate values ranging from $-56 \mathrm{dBm}$ to $-115 \mathrm{dBm}$ ). Moreover, we could only read RSSI information from neighboring base stations belonging to the same SIM card operator, constraining the practicality of this approach.

Accelerometer values from current state of the art smart phones are generally too noisy to be considered a reliable source to implement an inertial indoor navigation unit. Step counting could be an approach that we plan to further research in the future.

\section{CONLUSIONS}

We have developed an application for indoor localization to be implemented in current state of the art smart phones, leveraging their sensing capabilities in order to deliver up to 1.5 meters accuracy without the requirements for complex hardware that existing solutions need. In fact, to the best of our knowledge, our localization application is the first one delivering up to 1.5 meters resolution making use of only the hardware embedded within the phone and integrating both online and offline phases of RSSI fingerprinting within the same device. Additionally, we have implemented a new approach for the statistical processing of RSSI data in the phones, outperforming existing deterministic techniques.

\section{REFERENCES}

[1] Ryder, J.; Longstaff, B.; Reddy, S.; Estrin, D. 2009 Ambulation: A tool for monitoring mobility patterns over time using mobile phones, Proceedings - 12th IEEE Int. Conf., CSE 2009, v 4, 927-931.

[2] Shayeganfar, F.; Anjomshoaa, A.; Tjoa, A.; A smart indoor navigation solution based on building information model and google android, Lecture Notes in Computer Science, v 5105 LNCS, 1050-1056

[3] David, S.; Edlich, S. 2009 A location-based notificationAnd visualization-system indicating social activities, Proceedings of SPIE , v 7256, 2009.

[4] Xianhua, S.; Zhenjun, D.; Rong, C. 2009 Research on mobile location service design based on Android, Proceedings - 5th Int. Conf., WiCOM 2009.
[5] Li, X. 2009. Ratio-based zero-profiling indoor localization, IEEE 6th Int. Conf. MASS, 2009, 40-49.

[6] Hsu, C.; Yu, C. 2009. An Accelerometer based approach for indoor localization, Symposia and Workshops on UIC'09 and ATC'09 Conferences, 2009, 223-227.

[7] Pathanawongthum, N.; Cherntanomwong, P. 2009. Empirical evaluation of RFID-based indoor localization with human body effect", 15th Asia-Pacific Conf. on Communications, 2009, 479-482.

[8] Poovendran, R., Wang, C., Sumit R., 2006. Secure Localization and Time Synchronization for Wireless Sensor and Ad Hoc Networks, Springer, 2006.

[9] Singh, R., Gandetto, M., Guainazzo, M., Angiati, D., Ragazzoni., C., 2004. A novel positioning system for static location estimation employing WLAN in indoor environment, IEEE PIMRC, v. 3, 2004, 1762-1766.

[10] Brida, P., Cepel, P., and Duha, J. 2005, Geometric Algorithm for Received Signal Strength Based Mobile Positioning, Proc. Of Czech Slovak Technical Universities \& URSI, v. 5, 2005.

[11] Varshavsky, A., Lara, E., Hightower J., Lamarca, A., Otsason, V. 2007, GSM indoor localization, Pervasive and Mobile Computing, v. 3 , 6, 2007, 698-720.

[12] Otsason, V., Varshavsky, A., Lamarca, A., De Lara, E., 2005, Accurate GSM Indoor Localization, UbiComp 2005, 141-158.

[13] Roxin, A., Gaber, J., Wack, M., Nait-Sidi-Moh, A., 2007. Survey of Wireless Geolocation Techniques, Globecom Workshops, 2007, 1-9.

[14] Dempster, A., Li, B., Quader, I., 2008. Errors in deterministic wireless fingerprinting systems for localization, ISWPC 2008, 111-115.

[15] Mahtab A., Hien N., Yunye J., Wee-Seng S., 2007. Indoor Localization Using Multiple Wireless Technologies, MASS 2007, Oct. 2007, 1-8.

[16] Bahl, P., Padmanabhan, V., Balachandran, A., 2000. Enhancements to the RADAR user location and tracking system, Technical Report MSR-TR-00-12, Microsoft Research, Feb. 2000.

[17] Youssef, M., 2004. HORUS: A WLAN-Based indoor location determination system, Ph.D. Dissertation, University of Maryland, 2004.

[18] King, T., Kopf, S., Haenselmann, T., Lubberger, C., Effelsberg, W., 2006. COMPASS: A Probabilistic Indoor Positioning System Based on 802.11 and Digital Compasses, 1st WiNTECH, Sept 2006, 34-40.

[19] Ekahau, 2010. http://www.ekahau.com, April 2010.

[20] Noh, A.S.-I., Lee, W.J., Ye, J.Y., 2008. Comparison of the Mechanisms of the Zigbee's Indoor Localization Algorithm Software Engineering, 9th ACIS Int. Conf., Aug. 2008, 13 - 18. 